\title{
A Review on Semantic web based E-learning Applications
}

\author{
${ }^{*}$ M Vinay ${ }^{1}$, Dr. Deepaanand ${ }^{2}$ \\ ${ }^{I}$ Scholar, JJT University, Rajasthan, INDIA. \\ ${ }^{2}$ Department of Computer Science, CMR Institute of Technology, Bengaluru, INDIA. \\ Corresponding Author: M Vinay
}

\begin{abstract}
Today, the growth in the technological usage in people's life has triggered an increased use of internet in mobile devices among teachers and students in many institutions. They use the technologies not only for the social networking and personal work but also for the discovery of new knowledge to prepare them for the demand of the current industries and world. One of the most valuable innovation in the current technological era is the semantic web. It helps to perform a meaningful browsing, development well designed learning material and learning management system. This paper profiles an in-depth review on various research work on the usage of semantic web in e-learning, which will help to design an effective e-learning environment which uses semantic web in full capacity.
\end{abstract}

Indexterms: E-Learning, Semantic Web, Ontology, Semantic Web, Educational Resource

Date of acceptance: 15-07-2017

\section{INTRODUCTION}

THE invasion of digital technology in our day to day life has changed the attitude towards the usage of technologies in education field. Even though the acceptance and usage of technologies in pedagogy happened in a slow pace, students and teachers are using internet and applications in their computer or laptop or mobile devices not only for social networking and personal usage but also for browsing new information or finding more in-depth knowledge on an existing information. Many instance they use the technologies to find an elaborative and extended view on a topic or subject. They also use many applications and technologies for discovering and designing better learning materials. The increased usage of internet in learning has encouraged many educational institutions to develop e-learning environment. E-learning helps the students to learn the subject or a specific topic in their own pace and place. Many educational institutions have successfully implemented e-learning in their curriculum using web portals, Learning Management System (LMS), Course Management System (CMS) and Content Management System [1]. The usage of these application has helped many teachers to implement a flipped learning environment in their classroom, which helped them to save time for both teachers and students for practical and real life learning. The educational researchers have identified that the better usage of e-learning can improve the teaching and learning experience. One of the application in the recent year which got more focus from the educational researchers is semantic web in e-learning. Semantic web helps to perform a meaningful browsing, design better learning resources and learning environment. Many research has given a detailed in-sight on the usage of semantic web in e-learning. This paper gives a brief review on various research work on this field. The remaining paper is categorized into four sections. Section II give brief on the structure ofe-learning. Section IIIprofiles the structure of semantic web. Thena brief is given on previous research work is shown in the section IV. Finally, the paper is concluded in the section V.

\section{Structure of E-learning}

The most common structure of e-learning involves three parts, which are inter related. Teachers, contents and students. Figure 1 shows the basic parts of an e-learning structure [2].

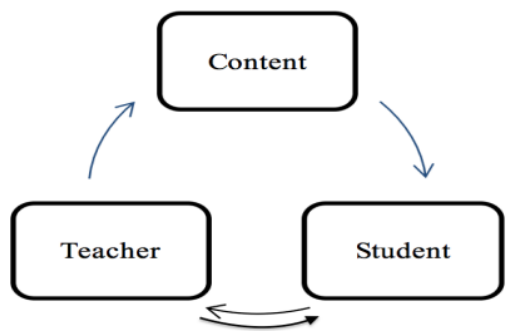

Fig.1. Basic parts of E-Learning structure 
The content is designed by teachers, which can be customized or designed based on the teacher's preference for the student's requirements. The most basic parts of most of the content designs are introduction, objective, sections of topics, assessments, summary and additional resources for the in-depth learning. Figure 2 shows the basic parts of a learning content design for e-learning.

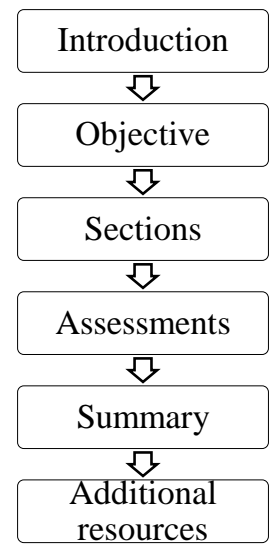

Fig.2. Basic parts of Content design for E-Learning structure

The e-learning structure is greatly influenced by the teachers, curriculum and the student's requirements. It can vary based on the institutions and their LMS and CMS. The semantic web can help in extracting the additional resource for the effective teaching learning environment. The semantic web can provide a personalized intelligent application to extract useful and better information related to each sections in the content design.

\section{Structure of Semantic web}

Semantic web is a compendium of standards, data structures and soft wares that helps to create an intelligent internet experience. Semantic web helps to extract meaningful data from internet. When semantic web is used in e-learning environment, it will help both teachers and students to gather meaningful and useful learning resources.

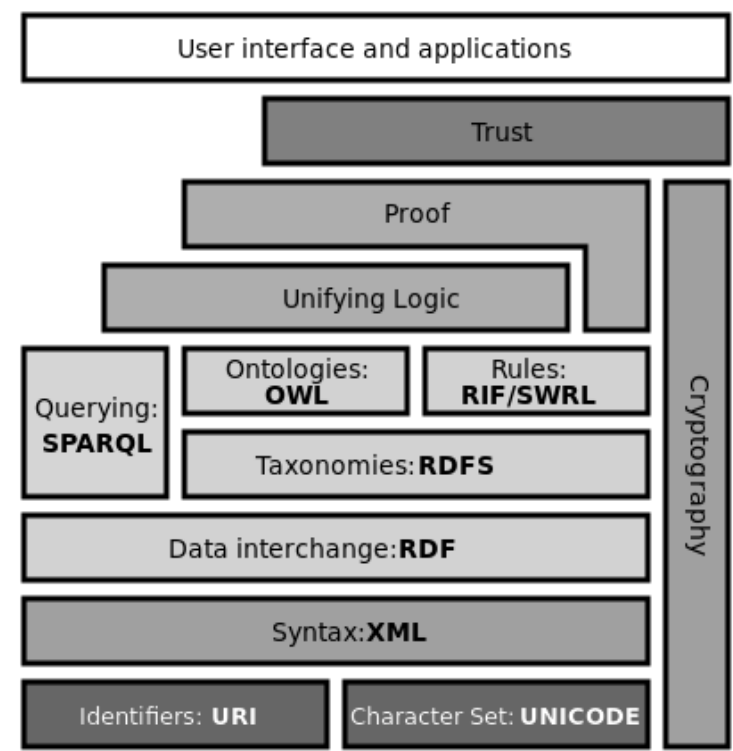

Fig. 3. Semantic Web stack [3]

Figure 3. shows the semantic web stack, which is the building blocks of an effective intelligent system powered with semantic web [3]. The character set is UNICODE that helps to define the standards for the representation of characters. The identifiers used is Uniform Resource Identifier (URI) and the syntax used is eXtensible Markup Language (XML). They are used for the data identification, description, conceptualization and categorization. The Resource Description Language, which includes Resource Description Framework (RDF), Resources Description Framework Schema (RDFS) and Rule Interchange Format (RIF) along with Web 
Ontology Language (OWL) and a semantic query language SPARQL helps to generate a language and structure for all the concept and ideas to relate to particular subject and topic and extract them accordingly [4].

\section{Related Work}

This section of the paper gives a brief review on previous research work in semantic web in e-learning from 2002 to 2016. Darell and Paul (2002) [5] has briefed about a system which can be used for performance based lifelong learning in their research work. According to the authors the system can help the computer system to extract the deep and exact details of the user's activities and their knowledge base.

Ronald and Michael (2003) [6] has described about a system which can be used as a process repository for the resource extraction of e-learning design in their research work. The system described $b$ he authors uses all the data from both development and production to form a semantic web developed with XML code base. The authors have used object oriented concepts in the system. Chitra, Vincent and Viswanath (2003) [7] has proposed a system which can extract educational resource using semantic web. According to the authors the system can automatically extract the video related the topic.

Yukari (2004) [8] has explained about a semantic web based mathematics learning intelligent application known as e-math interaction agent in his research work. According to the author the application can dynamically extract learning resources and related metadata framework to give a personalized learning experience for each user. Fabio, et al. (2004) [9] has presented a Learning Content Management System (LCMS) named as ROSA in their research work. According to the authors the application uses semantic web for content brows and storage. The application uses concept map in their application. Laura, et al. (2004) [10] performed a piolet study for the implementation of semantic web in e-learning in their research work. The authors used Material Property Data Markup Language (MatML) for the content delivery. The application also used a Materials Digital Library (MatDL) to collect the graphs by the users.

Juan, Luis and Martin (2005) [11] described about a web based brokerage system with a Reference Service Architecture which uses semantic web in their research work. The Authors explained about the ontology used in the system. They also discussed about the issues of intermediation system for e-learning in their research work. Ngamniji, Chidchanok and Peraphon (2005) [12] proposed an e-learning systemwhich uses a reference architecture in their research work. The authors used the help of web services and metadata-UDDI model in proposed system. According to authors they used XML data model for manipulating and expressing the metadata contents. Valentin (2005) [13] has presented an architecture and interface for an e-learning system with metadata registry in their research work.

Tao, et al. (2006) [14] explained an approach to use semantic web in e-learning in their research work. The authors also discussed about Educational Knowledge Service System (EKSS) prototype which helps to create, process, query and brows knowledge. Wenying and Deren (2006) [15] discussed about the method to integrate semantic web and ontology with e-learning in their research work. According to authors the method will help the users to select suitable course and retrieve knowledge efficiently. They also discussed about semantic querying and mapping approach.Changqin, et al. (2006) [16] presented a system called UACI-LR that is a secure Unified Access Control Infrastructure for e-Learning Resources management in their research work.

Wei and Xinmeng (2007) [17] discussed about the usage of ontology in e-learning in their research work. They discussed about a conceptual model which profiles the reusable contents in learning design and objects. They also discuss about the usage of ontology to showcase the concepts. Kazuo, et al. (2007) [18] proposed an e-learning tool which is a design engineering education framework in their research work. According to the authors the framework uses ShareFast. They uses semantic web to connect the design document and workflow. Chang-Yen and Wen-Ching (2007) [19] presented design for a service- oriented architecture for e-learning which uses semantic web to extraction, storage, retrieval and manipulation of the multimedia learning resources in their research work.

Chen and $\mathrm{Lu}$ (2008) [20] discussed about a e-learning model with semantic web services in their research work. They also discussed about CAS method in their work. Huang, Sui and Zhou (2008) [21] proposed an e-learning architecture in their research work. According to the authors the meaning of the learning resources and information exchange is defined using web service based on SOA and ontology in the proposed architecture. Yanyan and Mingkai (2008) [22] proposes a knowledge portal system for e-learning which uses semantic web for extraction, storage, retrieval and manipulation of knowledge in their research work.

Jamuna and Marie and Palanivel (2009) [23] discussed about a personalized e-learning system based on ontology and web services which maintain information exchange which is based on service-oriented architecture. Vibhvari (2010) [24] discussed an e-learning application using semantic web and proposed a conceptual architecture of the same in her research work. Iman and Sri (2011) [25] proposes an intelligent recommender system which uses semantic web for recommending relevant learning resources for the users in their research work. Joms, et al. (2012) [26] proposed an adaptive e-learning application which uses semantic web in their research work. The authors used an assessment based on short message services to create, store, 
retrieve and manipulate the knowledge base.Jianhua and Xuejun (2013) [27] discussed about an application which is an educational resource integration system with SOA and Web service in their research work. According to authors the system uses semantic web to discover and compose the knowledge. Foteini, Isidoros and Ioannis (2014) [28] proposed a methodology which uses semantic web to analyze and predict student's performance in their research work. According to the authors the system uses ontologies and semantic rules to enhance and deliver better educational contents.Shaimaa, Leandros and Francois (2015) [29] presented an approach for designing and implementing an ontology based learning management system in their research work. The authors discussed about a framework which uses students learning style and ontology to identify the impact of student's behavior. Herminio, Jose and MPuerto (2016) [30] described an e-learning tool based on semantic web to deliver better educational content in their research work. They also performed an experiment using the system among students to evaluate the performance and efficiency of the system.

\section{Conclusion}

Semantic Web has a great potential to create an effective e-learning environment. It can be integrated to e-learning platforms to design, develop, store and deliver better and effective educational resources. This paper discussed the basic structure of e-learning and semantic web. Then it gave a brief description on previous research work on semantic web in e-learning. The future work is to produce a better e-learning system powered by semantic web to give reliable teaching and learning platform for the teachers and students.

\section{References}

[1] Abazi-Bexheti, L. ; Apostolova-Trpkovska, M. \&Kadriu, A. "Learning Management Systems: Trends and Alternatives". 37 International Convention on Information and Communication Technology, Electronics and Microelectric (MIPRO). pp: 773-777. (2014).

[2] "E-Learning", Available at: http://etec.ctlt.ubc.ca/510wiki/Promoting_Success_In_E-Learning_For_The_High_School_Student

[3] Brewster, C. \& O'Hara, K. (2007), 'Knowledge representation with ontologies: Present challenges future possibilities', International Journal of Human-Computer Studies 65(7), pp. 563 - 568.

[4] "The semantic web and e-learning", Available at: http://elearnmag.acm.org/featured.cfm?aid=1555528.

[5] Woelk, Darrell, and Paul Lefrere. "Technology for performance-based lifelong learning." IEEE International Conference on Computers in Education,Proceedings., pp. 92-96, 2002.

[6] Hartwig, Ronald, and Michael Herczeg. "A process repository for the development of e-learning applications." The 3rd IEEE International Conference on Advanced Learning Technologies, 2003. Proceedings., pp. 346-347, 2003.

[7] Dorai, Chitra, Vincent Oria, and ViswanathNeelavalli. "Structuralizing educational videos based on presentation content." IEEE International Conference on Image Processing ICIP, Proceedings., vol. 2, pp. II-1029, 2003.

[8] Shirota, Yukari. "A metadata framework for generating web-based learning materials." IEEE International Symposium on Applications and the Internet Workshops SAINT Workshops, pp. 249-254, 2004.

[9] Porto, Fábio, A. Md C. Moura, and Fabio José Coutinho da Silva. "ROSA: a Repository of Objects with Semantic Access for eLearning." IEEE- Database, Engineering and Applications Symposium IDEAS'04, Proceedings. International, pp. 486-488, 2004.

[10] Bartolo, Laura M., Cathy S. Lowe, Adam C. Powell IV, Donald R. Sadoway, Jorge Vieyra, and Kyle Stemen. "Use of MatML with software applications for e-learning." Proceedings of the 4th ACM/IEEE-CS joint conference on Digital libraries, pp. 190-191, 2004.

[11] Santos, Juan M., Luis Anido, and Martín Llamas. "Design of a Semantic Web-based Brokerage Architecture for the E-learning Domain. A Proposal for a Suitable Ontology." 35th IEEE Annual Conference Frontiers in Education FIE'05, Proceedings, pp. S3HS3H., 2005.

[12] Arch-int, Ngamnij, ChidchanokLursinsup, and PeraphonSophatsathit. "A reference architecture for interoperating existing elearning systems using metadata and web services model." IEEE International Conference on Computational Intelligence for Modelling, Control and Automation And International Conference on Intelligent Agents, Web Technologies and Internet Commerce, vol. 2, pp. 891-896, 2005.

[13] Zacharias, Valentin. "A Metadata Registry For Community Driven E-Learning Sites." , IEEE International Conference on Computational Intelligence for Modelling, Control and Automation, And International Conference on Intelligent Agents, Web Technologies and Internet Commerce, vol. 2, pp. 871-876, 2005.

[14] Huang, Tao, Zongkai Yang, Qingtang Liu, Xia Li, Bei Wu, Sanya Liu, and Gang Zhao. "Semantic web-based educational knowledge service system (EKSS) for e-learning." First IEEE International Conference on Communications and Networking in China ChinaCom'06., pp. 1-5, 2006.

[15] Guo, Wenying, and Deren Chen. "Semantic Approach for e-learning System." First IEEE International Multi-Symposiums on Computer and Computational Sciences IMSCCS'06., vol. 2, pp. 442-446, 2006.

[16] Huang, Changqin, Yonghe Wu, Zhanjie Wu, and Zhiting Zhu. "A Unified Access Control Infrastructure Using Attributes and Ontology in E-Learning Resource Grids." IEEE International Conference on Computational Intelligence and Security, vol. 2, pp. 1481-1486, 2006.

[17] Yang, Wei, and Xinmeng Chen. "Applying Semantic web technologies to E-learning." IEEE International Conference on Wireless Communications, Networking and Mobile Computing WiCom, pp. 5415-5418, 2007.

[18] Hiekata, Kazuo, Hiroyuki Yamato, PiroonRojanakamolsan, and WataruOishi. "Design engineering educational framework using sharefast: a semantic web-based e-learning system." , Fourth IEEE International Conference on Information Technology ITNG'07, pp. 317-322, 2007.

[19] Lai, Chang-Yen, and Wen-Ching Liou. "A service-oriented architecture for constructing ontology-based learning objects repository." Ninth IEEE International Symposium on Multimedia Workshops ISMW'07., pp. 349-355. IEEE, 2007.

[20] Jing, Chen, and Lu Quan. "A complex adaptive E-learning model based on semantic web services." IEEE International Symposium on Knowledge Acquisition and Modeling KAM'08., pp. 555-559, 2008.

[21] Lan, Huang, Sui Ling-ge, and Zhou Chun-guang. "Research on the Sharing e-learning Based on SOA and Semantic Web Architecture." IEEE International Conference on Computer Science and Software Engineering, vol. 5, pp. 426-429, 2008. 
[22] Li, Yanyan, and Mingkai Dong. "Towards a Knowledge Portal for E-Learning based on semantic Web." Eighth IEEE International Conference on Advanced Learning Technologies ICALT'08., pp. 910-912, 2008

[23] Jamuna, Rani S., Marie Stanislas Ashok, and K. Palanivel. "Adaptive content for personalized E-learning using web service and semantic web." . IEEE International Conference on Intelligent Agent \& Multi-Agent Systems IAMA, pp. 1-4, 2009.

[24] Pandit, VibhavariRambhau. "E-Learning System Based on Semantic Web." 3rd IEEE International Conference on Emerging Trends in Engineering and Technology (ICETET), pp. 559-564, 2010.

[25] Paryudi, Iman. "Proposed intelligent e-learning system using semantic web." IEEE International Conference on Advanced Computer Science and Information System (ICACSIS, pp. 133-136, 2011.

[26] Antony, Joms, Fr Rubin Thottupuram, Shiney Thomas, and Melbin Varghese John. "Semantic web based adaptive e-learning triggered through short message services." 7th IEEE International Conference on Computer Science \& Education (ICCSE), pp. 1860-1863, 2012.

[27] Li, Jianhua, and Xuejun Wang. "To Discover and Integratethe Education Resources Based on Semantic Web." Fifth IEEE International Conference on Measuring Technology and Mechatronics Automation (ICMTMA), pp. 1264-1267, 2013.

[28] Grivokostopoulou, Foteini, IsidorosPerikos, and IoannisHatzilygeroudis. "Utilizing semantic web technologies and data mining techniques to analyze students learning and predict final performance." IEEE International Conference on Teaching, Assessment and Learning (TALE), pp. 488-494, 2014.

[29] Nafea, Shaimaa M., Leandros A. Maglaras, Francois Siewe, and M. ElemamShehab. "A novel adaptive learning management system using ontology." IEEE Seventh International Conference on Intelligent Computing and Information Systems (ICICIS), pp. $328-335,2015$

[30] Garcia-Gonzalez, Herminio, Jose Emilio Labra Gayo, and MPuertoPaule-Ruiz. "Enhancing e-Learning content by using Semantic Web technologies." IEEE Transactions on Learning Technologies (2016).

IOSR Journal of Computer Engineering (IOSR-JCE) is UGC approved Journal with Sl. No. 5019, Journal no. 49102.

M Vinay. "A Review on Semantic web based E-learning Applications." IOSR Journal of Computer Engineering (IOSR-JCE) 19.4 (2017): 34-38. 